\title{
Faire l'expérience de soi
}

\author{
Note de lecture \\ Wolfgang Iser, L'appel du texte. L'indétermination comme condition d'effet \\ esthétique de la prose littéraire, trad. V. Platini, Paris, Allia, 2012, 60 pages
}

\section{Martine BÉlAnd}

Avec L'appel du texte, les éditions Allia, que les amateurs d'essais en véritable format poche connaissent bien, publient le second livre de Wolfgang Iser (1926-2007) en traduction française. Malgré l'intérêt de la théorie littéraire et de l'herméneutique philosophique pour les idées de l'école de Constance et du groupe de recherche Poétique et herméneutique (1963-1994) — fondé notamment par Iser, Hans-Robert Jauss et Hans Blumenberg —, les lecteurs francophones devaient jusqu'ici se satisfaire d'un seul livre d'Iser (L'acte de lecture. Théorie de l'effet esthétique, traduit en 1985), dont les idées ont pourtant beaucoup influencé le champ des études littéraires en Europe comme aux États-Unis ${ }^{1}$. Jauss, plus présent en français ${ }^{2}$, a lui-même dit que les projets mis en chantier par «l'école de Constance » et «le tournant vers une théorie de la réception et des effets » ont été lancés par son Histoire de la littérature comme provocation (1967) ainsi que par L'appel du texte d'Iser (cité in Kalinowski 152).

L'appel du texte est le discours inaugural présenté par Iser à l’Université de Constance en 1969 sous le titre Die Appellstruktur der Texte, et publié en allemand l'année suivante. Ce texte précède donc les œuvres maîtresses qu'Iser a fait paraître en 1972 (Der implizite Leser, inédit en français) et en 1976 (L'acte de lecture), lesquelles ont poursuivi l'exploration des pistes ouvertes 
en 1969. Aussi le lecteur ne doit-il pas être étonné de constater que dans ce court essai, Iser évoque des tâches qu'il ne remplit pas ici (Iser, Appel 33), qu'il emploie fréquemment le mode conditionnel (39, etc.) ou qu'il suggère (mais sans plus) des idées devenues centrales quelques années plus tard (comme celle du «lecteur implicite », par exemple [47]). Car on est bien face, ici, à un programme de travail, où Iser propose des intuitions fondamentales et esquisse quelques thèses qui orienteront ses recherches au cours des années suivantes.

D’entrée de jeu, Iser pose une question directrice : «[C]omment peut-on décrire la relation qui lie texte et lecteur? » (12) Cette question, qui oriente tout le travail d'Iser, ne trouve pas de réponse complète dans cet essai qui vise, avant tout, à baliser les conditions de possibilité d'une telle enquête descriptive. La théorie littéraire doit dorénavant tourner son attention vers le lecteur plutôt que vers l'auteur: l'étude des fonctions internes du texte ne peut se passer d'examiner les interactions entre le texte et le lecteur, de façon à interroger les effets du premier sur le second. Sa thèse est claire : «[L]es significations des textes littéraires sont avant tout engendrées dans le processus de lecture »(11). Elle s'appuie sur une évidence : si le sens du texte est le produit de la relation entre le texte et le lecteur, c'est bien parce que le texte est actualisé par la lecture. C'est à un véritable «tournant» dans les études littéraires qu'Iser appelait ainsi en 1969; mais aussi, il proposait par là même un chantier nouveau pour l'esthétique au sens large et pour l'anthropologie $(59-60)^{3}$. Le champ littéraire, d'ailleurs, était prêt à prendre ce tournant, comme en témoigne l'importance qu'a prise, notamment aux États-Unis, la readerresponse theory ${ }^{4}$ qui, chez Stanley Fish, Norman N. Holland ou Robert C. Holub, s'est attachée à décrire et à théoriser le « retour » du lecteur ( $c f$. Freund) dès l'aube des années 1970.

En s'ouvrant sur une citation du Against Interpretation (1966) de Susan Sontag invitant à «l'éveil des sens» en art (Iser, Appel 7), et se clôturant sur l'importance de «l'expérience de 
soi » selon le poète élisabéthain Philip Sidney (60), L'appel du texte montre clairement qu'il s'agit, dans l'esthétique isérienne, de s'éloigner de l'herméneutique ou de l'art de l'interprétation (il critique ici le germaniste suisse Emil Staiger, 1908-1987), pour scruter, comprendre et théoriser plutôt l'effet esthétique des œuvres écrites. La condition de cet effet est l'indétermination de l'œuvre, comme l'annonce déjà le sous-titre de cette leçon inaugurale. «[C]ette indétermination constitue une condition élémentaire d'effets textuels » (13), ce que les trois parties centrales du discours de 1969 visent à démontrer. Iser suit donc trois étapes pour esquisser la relation texte/lecteur en ce qui a trait au texte littéraire ${ }^{5}$ : d'abord, par une analyse extérieure, il tente de cerner la particularité du texte littéraire (15-21); ensuite, par une analyse intérieure ou formelle, il s'attache à analyser les conditions élémentaires des effets qu'il produit, ainsi que les différents degrés d'indétermination qui lui sont propres (22-40); enfin, par de brèves études de cas, il tâche d'expliquer l'accroissement de son degré d'indétermination depuis le XVIII ${ }^{\mathrm{e}}$ siècle (41-55). On peut résumer brièvement ces trois étapes de son enquête.

En ce qui a trait, d'abord, à la spécificité du texte littéraire, Iser précise qu'il s'agit de sa dimension performative ${ }^{6}:$ le texte littéraire engendre son objet, contrairement aux textes constatatifs (15) ou d'exposition (18). La réalité propre au texte littéraire est celle qu'il constitue lui-même : il est une «réaction » (18) et « une mise à disposition des points de vue » (17) sur la réalité quotidienne. C'est pourquoi le rôle du lecteur est essentiel : le texte littéraire n'engendre pas d'objet «véritable»: «[I]1 n'acquiert sa réalité effective que par l'opération du lecteur réagissant à ce que le texte lui propose » (18). Le texte n'est que pour autant qu'un lecteur réponde à son appel. C'est pourquoi l'expérience personnelle du lecteur est capitale, ainsi que le préciseront la troisième partie et la conclusion du discours d'Iser : comme on ne peut pas sortir du texte littéraire pour vérifier son objet (comme c'est le cas, au contraire, pour le texte 
d'exposition), le lecteur «n'a que sa propre expérience personnelle pour se forger une opinion sur ce qui lui est communiqué »(18). Iser insiste à plusieurs reprises sur le fait que «nos expériences personnelles jouent un rôle considérable dans la réalisation des textes »— de telles affirmations renforcent certes l'importance de «l'objet lecteur » pour le théoricien du littéraire —, mais il prend soin d'ajouter qu'à travers le processus de lecture, « il advient toujours quelque chose de nos expériences » (19). Autrement dit, la relation texte/lecteur est à deux sens : le texte s'actualise (autrement dit, concrétise son sens possible) à travers la lecture, et le lecteur fait ainsi l'expérience du texte et l'expérience de lui-même (cf. 60). Mais tout lecteur de roman n'est-il pas justement conscient de se retrouver lui-même à l'œuvre dans le texte littéraire? Et lorsqu'on abandonne la lecture d'une œuvre en cours de route, ne le fait-on pas parce que cette œuvre ne nous a pas interpellés, qu'elle ne nous a pas donné la possibilité de nous y retrouver, de communiquer avec elle? En tentant ainsi de théoriser des expériences ressenties quotidiennement et immédiatement par tout lecteur, Iser travaille en phénoménologue, comme il l'a lui-même noté plus tardivement ${ }^{7}$, ce qui n'est pas dépourvu de difficultés théoriques.

La spécificité du texte littéraire repose donc sur sa «capacité à osciller »(Iser, Appel 21) entre la réalité objective du Lebenswelt et l'expérience personnelle du lecteur. Si le texte littéraire est «performatif », c'est parce que le texte et sa lecture sont un mouvement: vers le lecteur et vers le texte. La lecture est un acte qui répond à l'appel du texte indéterminé. Quelles sont donc, dans le texte littéraire même, les conditions à l'origine de son indétermination? Cette question ouvre la deuxième partie de l'essai d'Iser, qui est en quelque sorte placée sous le patronage de Roman Ingarden. Iser reprend ici l'idée d'« aspects schématisés » ${ }^{8}$ pour construire sa conception du texte littéraire comme étant une multiplicité de «points de vue » (22) ou de «facettes» (23), qui implique l'«ouverture» (24, note) de l'œuvre et, de fait, son 
indétermination. C'est en raison de cette multiplicité et de cette ouverture que le texte littéraire présente des «seuils de liberté » (32) (Iser parle aussi de «vides » [24]) au lecteur qui peut alors l'interpréter et lui donner un sens. Or, si l'on revient, un instant, à la question initiale d'Iser dans cette partie — à savoir : d'où provient l'indétermination du texte? —, il est difficile de ne pas ressentir une nette impression de circularité : Iser n'est-il pas en train de proposer que la condition de l'indétermination du texte est la multiplicité de ses points de vue, à savoir son indétermination? L'analyse formelle, ici, demande à être complétée par les développements qui suivront en 1972 et en 1976 dans Le lecteur implicite et L'acte de lecture, ainsi que par le débat qui s'est ouvert en 1981 entre Stanley Fish et Iser sur cette question de la nature et de l'effet de l'indétermination ou du « non-dit » ( $c f$. Heidenreich $80 s q q$.).

Mais reprenons le fil du discours de 1969. Iser affirme que les vides — ou l'indétermination — sont « les prémices » (26) à l'effet esthétique, puisque ce sont eux seuls qui «permettent au lecteur de participer à la constitution du sens » (27). C'est pour Iser une autre manière de dire que «la lettre du texte n'épuise pas son intention» (38), ce que bien des romanciers et littéraires ont compris depuis longtemps. Iser le théoricien se fait d'ailleurs pédagogue en montrant que c'est en puisant dans la lecture d'œuvres littéraires que l'on peut comprendre leurs fonctions formelles et leurs effets externes. Il renvoie ainsi à une affirmation épistolaire du romancier britannique William M. Thackeray (1811-1863): «[I]t is the unwritten part of books that would be the most interesting » (47, note). Iser s'en inspire pour avancer que dans le texte littéraire, "[1]e plus important est passé sous silence » : l'intention du texte n'est pas dans le texte, mais bien « [d]ans l'imagination du lecteur » (57) qui « coopère » avec le texte et y «participe » (27) — au point qu'Iser propose une formule importante, qui marque le départ de la théorie littéraire loin des rives auctoriales traditionnelles : le lecteur serait un «coauteur» 
du texte littéraire (31). Iser s'en remet ici à l'étude de cas pour démontrer que les littéraires euxmêmes étaient déjà conscients de cette imbrication des pôles de la production et de la réception. Il se penche sur le cas particulier du roman-feuilleton — extrêmement courant et fort apprécié au $\mathrm{XIX}^{\mathrm{e}}$ siècle - et de la technique de découpage que cette pratique éditoriale a développée. Ainsi Charles Dickens, par exemple, tentait d'apprendre « comment les lecteurs s'imaginaient la suite de l'intrigue » avant de l'écrire (29). En créant des attentes et en suscitant l'imagination du lecteur par rapport aux péripéties, le roman-feuilleton invitait expressément le lecteur à prendre «plus largement part au déroulement de l'histoire » (31). Dans ce cas, l'interpellation du lecteur par le texte était clairement revendiquée, ressentie et connue de part et d'autre.

L'exemple du roman-feuilleton permet aussi à Iser de souligner que le degré d'indétermination des textes littéraires a beaucoup varié dans l'histoire de la littérature, et qu'il s'est considérablement accru au cours des trois derniers siècles. Dans la troisième partie de son discours, Iser montre cet accroissement en s'arrêtant à trois cas issus de la littérature anglaise : Henry Fiedling (1707-1754), Thackeray et le Ulysses de James Joyce (1882-1941). Il souligne que l'indétermination est si grande chez Joyce, que le texte confronte le lecteur à ses propres attentes et l'invite à comprendre ses propres représentations (53). En effet, «[a]u degré élevé d'indétermination répond une projection massive de signification » (53). Plus un texte littéraire est indéterminé et ouvert, plus le lecteur y mettra de lui-même. L'idée qui a eu un rapide effet en théorie littéraire est précisément celle qui permet de lier cette indétermination du texte au titre du discours de 1969 — ou du moins, à son titre allemand: la structure d'appel du texte. Iser propose que le texte écrit «ne serait que l'ombre diffuse » (48) de son fondement implicite, à savoir de l'indétermination, lequel fondement implique nécessairement une relation texte/lecteur et un échange entre eux : le texte est une structure d'appel, un appel fait au lecteur pour qu'il 
engendre la signification que le texte peut avoir. «L'indétermination enclenche l'imaginaire du lecteur et l'engage à accomplir l'intention dont le texte est dépositaire » — cette intention étant de faire sens. Iser poursuit : «Cela signifie aussi que l'indétermination est au fondement d'une structure textuelle qui prend toujours et préalablement le lecteur en ligne de compte » (56).

C'est dans la péroraison que se dessine la dimension plus largement philosophique de l'esthétique de la littérature dessinée par Iser en 1969. Iser, après tout, affirmait d'entrée de jeu que si son champ propre était le littéraire, la portée de sa théorie était beaucoup plus large. En considérant « les rapports ici débattus entre texte et lecteur comme un possible préambule à ce problème de la condition humaine » (14), il s'agissait d'interroger la menschliche Situation. Iser donnait donc d'emblée à ses recherches une dimension philosophique. Il considérait d'ailleurs la question finale de L'appel du texte - pourquoi lit-on encore? (59) - comme une question proprement anthropologique. En guise d'amorce à une théorie - qu'il élaborera vingt ans plus $\operatorname{tard}^{9}$ - Iser avançait que la lecture permet une prise de «conscience de soi-même » (59), une expérience de soi dans la liberté de la réflexion, liberté rendue possible par l'indétermination du texte. L'indétermination est donc « un élément crucial de la fonction effective de l'œuvre d'art » en ce sens qu'elle « conditionne » la réception du texte (24, note), mais aussi en ce sens qu'elle rend possible la formation de soi, la Bildung dont l'idéal pédagogique irrigue la culture occidentale. C'est d'ailleurs parce que l'intention du texte littéraire se trouve dans l'imagination du lecteur et non pas dans le texte lui-même que ce dernier peut « résister aux siècles » (57) : car sa structure permettra toujours au lecteur de s'impliquer dans la réalisation de son sens.

Iser rappelle que l'élément primordial d'un texte littéraire est d'être lu (56). En ce sens, ne pouvait-il pas déjà étendre la portée de sa thèse sur l'échange entre le texte et le lecteur bien au-delà du roman? En effet, on pourra toujours trouver plaisir et sens à lire du Cervantes ou du 
Balzac, mais aussi du Descartes, du Platon ou du Nietzsche. Car la force et la fonction du texte philosophique ne se trouvent pas uniquement dans sa prétention scientifique (heureusement, d'ailleurs, car sinon, pourquoi lirait-on encore Aristote?), mais aussi dans sa dimension esthétique ou performative. Et même un texte «savant » (qu'il s'agisse d'un livre de philosophie ou d'un essai d'astrophysique) dépend du lecteur pour l'effet de son sens. Que l'objet du texte lui soit extérieur ou pas ne change rien au fait que le texte ne dit rien et ne fait rien si personne ne le lit. Certes, les astres n'attendent pas qu'on lise un manuel sur les lois de leur mouvement. Mais la lecture d'un tel manuel entraîne des effets culturels et sociaux. Friedrich Nietzsche, dont les livres ne trouvaient pas de lecteurs à leur parution, était amèrement conscient de ce fait. Il posait ce constat en 1885 : «Mes livres sont des hameçons; s'ils n'attirent personne vers moi, alors ils n'ont aucun sens! » (lettre à Förster-Nietzsche de la mi-août 1885). Il voyait que faute de lecteur, « [s]a littérature n'exist[ait] plus » (lettre à Overbeck du début décembre 1885). Le théoricien de la littérature Gérard Genette a bien souligné qu'un livre que personne ne lit est condamné à disparaître : «[U]n livre mal lu, et a fortiori non lu à sa première édition risque de n'en pas connaître d'autre » (Genette 183). Or, au-delà des questions purement éditoriales, c'est aussi le contenu et ses effets possibles qui sont ainsi condamnés à disparaitre, puisque la réalisation effective du sens d'un texte dépend de sa lecture. La leçon inaugurale d'Iser présentait donc déjà des bases utiles pour considérer le fondement littéraire - entendre ici esthétique — de tout texte et de tout rapport à un texte. Car la définition du texte donnée au tout début de L'acte de lecture en 1976 — à savoir que «[1]e texte est un potentiel d'action qui est actualisé au cours du processus de lecture » (Iser, Acte 4) — vaut bien pour tout texte. Autrement dit, les thèses dessinées dans L'appel du texte présentent les bases d'une théorie de la lecture qui s'oriente vers 
une anthropologie esthétique, dépassant de beaucoup les visées immédiates d'une théorie du roman.

L'écriture, disait Nietzsche, témoigne d'une « expérience vécue » par l'auteur et invite le lecteur à faire une telle expérience pour lui-même (Nietzsche, «Préface » § 1). La matérialité du texte (le livre, l'article, comme objets) ne doit donc pas nous induire à considérer le texte comme étant un objet terminé. À cet égard, Iser était proche de son collègue Jauss, bien sûr — selon qui « la concrétisation du sens de l'œuvre » dépend des deux «éléments constitutifs » que sont l'effet (ou l'action) de l'œuvre et la réception de celle-ci par un destinataire (Jauss 284) —, mais aussi de Jacques Derrida qui reconnaissait l'ouverture de l'œuvre : « Nous savons la saturation sémantique impossible » (Derrida 27). En lisant, on interprète; en interprétant, on « sélectionne » (51); en sélectionnant, on «dévoile beaucoup de [nous]-même » (Iser Appel 48). La leçon inaugurale d'Iser invitait donc son public à développer une pratique de la lecture comme exercice conscient d'expérience et de construction de soi. C'est en raison de cette dimension performative et réflexive que ses idées pourraient faire leur incursion dans la méthodologie philosophique. 


\section{Notes}

${ }^{1}$ Au moment de recevoir Iser, en 2000, dans le cadre des Stanford Presidential Lectures in the Humanities and Arts, David Albertson écrivait : "Iser's work continues to exert a far-reaching, if quiet influence in literary studies. Many students (and scholars), not unlike apprentices in the master artisan's studio, are scarcely aware of the existence of the influence, much less the source. And yet however quietly, Wolfgang Iser undoubtedly stands among the most prominent literary theorists of the late twentieth century »(Albertson). On peut par ailleurs rappeler la publication, en français, d'un court texte d'Iser dans la revue Poétique en 1979.

${ }^{2}$ La publication des deux œuvres maîtresses de Jauss chez Gallimard (et donc la diffusion que cela implique) n'y est certainement pas étrangère. En comparaison, le livre d'Iser fut publié en Belgique, aux éditions spécialisées (et peu diffusées) Mardaga.

${ }^{3}$ Sur la dimension anthropologique du projet d'Iser, on peut lire l'entrevue qu'il a accordée à la University of California at Irvine en 1998, publiée dans la revue Anthropoetics, "The Use of Fiction ».

${ }^{4}$ Les théoriciens américains distinguent clairement entre la reader-response theory et l'étude de réception, ce qui recoupe - mais en les confondant parfois - la distinction allemande entre l'étude de l'effet (Wirkungsgeschichte) et l'esthétique de la réception (Rezeptionsästhetik). Cf. par ex. l'essai de Patricia Harkin sur «la réception de la reader-response theory» (!) : «To begin, I need to discriminate reader-response theory from reception study [...]. I use the term "reception study" to refer to an inquiry into a text's effect on specific classes of readers (women, members of the working class in Liverpool, and so forth). [...] Reader-response theory, by contrast, is properly an effort to provide a generalized account of what happens when human beings engage in a process they call "reading" 》 (Harkin 411). Par-delà les « antagonismes théoriques » au sein de l'esthétique de la réception (Kalinowski 151 sq.), on peut certainement affirmer minimalement que les théoriciens de la réception, de l'effet et de l'acte du lecteur se réclament d'une « rupture avec l'esthétique traditionnelle de la production et le "changement de paradigme" qui place le lecteur au centre de la théorie littéraire » (151).

${ }^{5}$ Comme le remarque Rosmarin Heidenreich, Iser applique son modèle exclusivement au roman (Heidenreich 79). Il suggérait pourtant, vers la fin de son discours de 1969, pouvoir l'élargir à la « littérature en général » (Iser, Appel 56).

${ }^{6}$ Iser (Appel 15 sqq.) renvoie ici aux théories d'Austin, Quand dire c'est faire; $c f$. aussi Iser, Acte $15 s q$.

${ }^{7}$ La dimension «phénoménologique » du travail d'Iser repose en ceci qu'il examine l'œuvre en tant qu'elle apparaît à travers une expérience, celle de la lecture : «The phenomenological theory of art lays full stress on the idea that, in considering a literary work, one must take into account 
not only the actual text but also, and in equal measure, the actions involved in responding to that text $\gg$ (Iser, Reading Process 279). Ici, Iser se réclame une fois de plus d'Ingarden.

${ }^{8}$ Iser renvoie à L'euvre d'art littéraire, publié par Ingarden en 1960 (et en français en 1983). Notons aussi qu'alors qu'Ingarden parlait de « polyvalence », Iser parle de «polyphonie » (Iser, Appel 25, note) : les échos d'Ingarden chez Iser sont ici nombreux, et parfois de manière critique.

${ }^{9}$ Iser a développé son « anthropologie » littéraire dans son ouvrage de 1991, Das Fiktive und das Imaginäre. Perspektiven literarischer Anthropologie. On voit très bien, ne serait-ce que par le titre de cet ouvrage, que l'anthropologie isérienne trouve son point de départ dans les idées avancées en 1969 autour de la fusion entre l'indétermination de l'œuvre et l'imagination du lecteur.

\section{Textes cités}

Albertson, David, Présentation de Wolfgang Iser, Stanford Presidential Lectures in the Humanities and Arts, Université Stanford, 2000, texte en ligne: http://prelectur.stanford.edu/lecturers/iser/ (page consultée le 4 sept. 2013).

FREUnD, Elizabeth, The Return of the Reader. Reader-Response Criticism, Londres/New York, Methuen \& Co., 1987.

Genette, Gérard, Seuils, Paris, Seuil, 1987.

HARKIN, Patricia, «The Reception of Reader-Response Theory », College Composition and Communication, vol. 56, $\mathrm{n}^{\circ}$ 3, 2005, p. 410-425.

HEIDENREICH, Rosmarin, «La problématique du lecteur et de la réception », Cahiers de recherche sociologique, vol. 12, 1989, p. 77-89.

ISER, Wolfgang, L'acte de lecture. Théorie de l'effet esthétique, trad. E. Sznycer, Bruxelles, Mardaga, coll. Philosophie et langage, 1985.

—, L'appel du texte. L'indétermination comme condition d'effet esthétique de la prose littéraire, trad. V. Platini, Paris, Allia, 2012.

—, « La fiction en effet », Poétique, n³9, 1979, p. 275-298.

—, Der implizite Leser. Kommunikationsformen des Romans von Bunyan bis Beckett, Munich, Fink, 1972.

—, «The Reading Process. A Phenomenological Approach », New Literary History, vol. 3, n 2, 1972, p. 279-299. 
—, « The Use of Fiction in Literary and Generative Anthropology. An Interview with Wolfgang Iser », entrevue par Richard van Oort, Anthropoetics. The Electronic Journal of Generative Anthropology, vol. 3, $\mathrm{n}^{\circ} 2$ (1998), texte en ligne :

www.anthropoetics.ucla.edu/ap0302/Ap0302.htm (page consultée le 6 sept. 2013).

JAUSS, Hans Robert, «L'esthétique de la réception : une méthode partielle », postface à « De l'Iphigénie de Racine à celle de Goethe », in Jauss, Pour une esthétique de la réception, trad. C. Maillard, Paris, Gallimard, coll. Tel, 1978, p. 266-287.

KALINOWSKI, Isabelle, «Hans-Robert Jauss et l'esthétique de la réception. De “L'histoire de la littérature comme provocation pour la science de la littérature" (1967) à "Expérience esthétique et herméneutique littéraire" (1982) », Revue germanique internationale, vol. 8, 1997, p. 151-172.

NIETZSCHE, Friedrich, "Préface à la deuxième édition », in Nietzsche, Le gai savoir, dir. G. Colli et M. Montinari, trad. P. Wotling, aris, GF, 2007, p. 25-33.

-, Sämtliche Briefe. Kritische Studienausgabe, dir. G. Colli et M. Montinari, Berlin, de Gruyter/DTV, 8 vol., 2003. 